\title{
DISPERSION CHARACTERISTICS OF HOLLOW-CORE PHOTONIC CRYSTAL FIBERS
}

\author{
I. A. Khromova ${ }^{1}$, L. A. Melnikov ${ }^{2}$ \\ Saratov State University, Saratov, Russia
}

This work is devoted to the study of hollow-core photonic crystal fibers (HCPCF) dispersion properties. An effective scheme of HCPCF eigenmodes analysis, lying in separate dispersion diagrams calculation for the cladding and for the HCPCF itself, was proposed. General recommendations concerning selection of proper fiber parameters were given.

Работа посвящена изучению дисперсионных свойств фотонно-кристаллических волноводов с полой сердцевиной (ПФКВ). Предложена эффективная схема анализа собственных мод ПФКВ, в основе которой лежит расчет дисперсионных диаграмм оболочки (решетки) и волокна (сверхрешетки) в рамках отдельных задач. Даны общие рекомендации по выбору оптимальных параметров волноводов.

\section{INTRODUCTION}

In recent years photonic crystals attract more and more attention of scientists and technologists as they hold much promise in terms of the variety of their applications [1]. These objects are famous for unique and tunable dispersion properties and for the existence of so-called photonic band gaps, i.e., regions of frequencies at which light propagates in the photonic crystal medium. The idea of using this effect as an analogue of total internal reflection resulted in the developement of a new sort of waveguides: hollow-core photonic crystal fibers (HCPCF) [2-4], i.e., photonic crystal fibers with air-filled core. The photonic band gap effect in the HCPCF cladding leads to light being confined within the core, with only about $5 \%$ of light intensity fall at glass cover structure, which implies low nonlinear effects. This fact makes HCPCF advantageous for high power pulse transmission when compared to convenient fibers [5]. Selecting different geometrical structure, material and core shape, and filling the hollow cores with gases allow one to control the HCPCF dispersion and nonlinear properties and use them to solve a wide variety of problems $[6,7]$.

Theoretically predicted low losses of HCPCF systems (in [8] they are reported to be about $0.02 \mathrm{~dB} / \mathrm{km}$ ) make them very promising in terms of application in optical communication. At present, the losses in real systems are almost hundred times larger than those estimated in [8]. It is a result of light scattering at core walls and structure period irregularity and

\footnotetext{
${ }^{1}$ E-mail: KhromovaIA@yandex.ru

${ }^{2}$ E-mail: lam@sgu.ru
} 
nonoptimal way of theoretical parameter selection for different values of wavelengths. The former reasons are evidently problems of technology and can be solved only by improving the corresponding manufacturing equipment, whereas optimizing of HCPCF characteristics at its design stage involves developing a general theoretical approach to light propagation in such structures. This paper reports on the work devoted to the study of HCPCF dispersion properties and development of general mode and parameter selection algorithm for efficient light transmission.

\section{HCPCF MODES CALCULATION}

Plane Wave Method. In order to obtain the fiber modes spectrum, we calculated the dispersion diagrams for the cladding of the fiber (as a two-dimensional photonic crystal) and for the fiber core (representing the HCPCF as a superlattice photonic crystal). Comparing these diagrams allows one to analyze the waveguiding properties of the fiber.

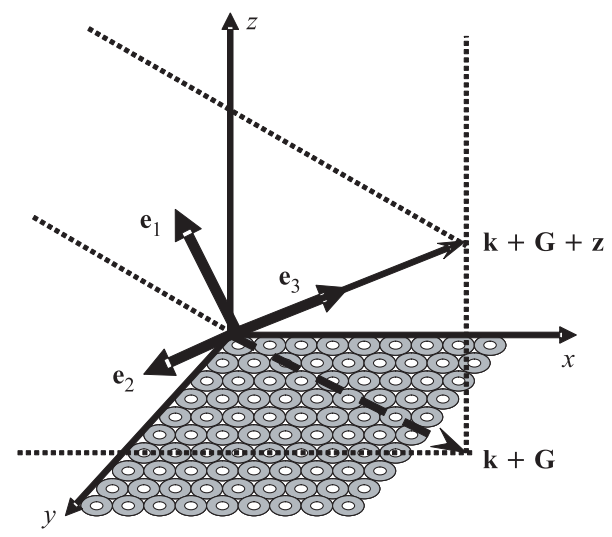

Fig. 1. Plane wave method coordinates. Here $\left(\mathbf{e}_{1}, \mathbf{e}_{2}, \mathbf{e}_{3}\right)$ is the plane wave decomposition basis, $z$ is the HCPCF axis, $\mathbf{k}$ is the transversal wave vector, $\beta$ is the propagation constant, $\mathbf{G}$ is the cladding reciprocal lattice vector

Figure 2 shows an example of an HCPCF with a diamond-shaped core made of $B K 7$ glass (with refraction index $n=1.52$ ) with the cladding period $d=4.6 \mu \mathrm{m}$, the walls thickness $\Delta=300 \mathrm{~nm}$ and the core diameter $D \approx 300 \mu \mathrm{m}$. All the results given below were obtained for this very structure.

To solve the dispersion equations for the cladding, we used the modification of the plane wave method [3-5] with the wave-oriented coordinate system (see Fig. 2), allowing one to operate a real symmetric equation matrix.

Using this method, we have studied the properties of the cladding, namely, the influence of material and geometric properties (like refraction index contrast, periodicity, etc.) on the dispersion ones. We have evaluated the approximate shifts of band gaps and it appeared that, for instance, for the HCPCF shown in Fig. 2 a $1 \%$ change in the periodicity of the cladding results in a shift of the structure band gap. 
302 Khromova I. A., Melnikov L.A.

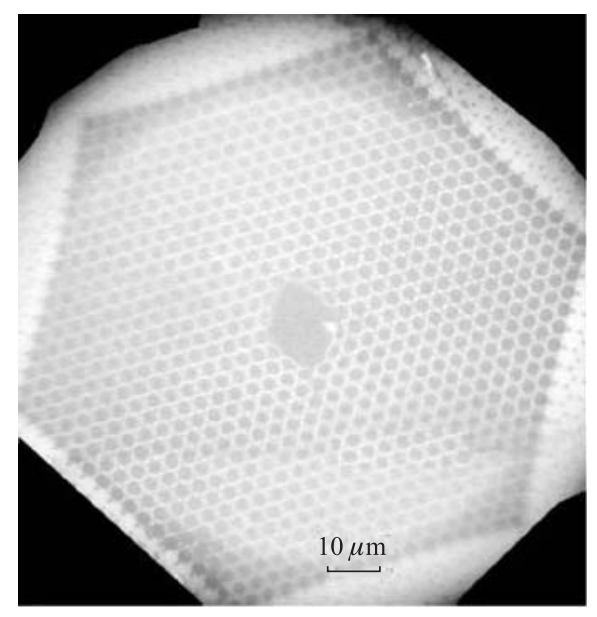

Fig. 2. Diamond-shaped HCPCF
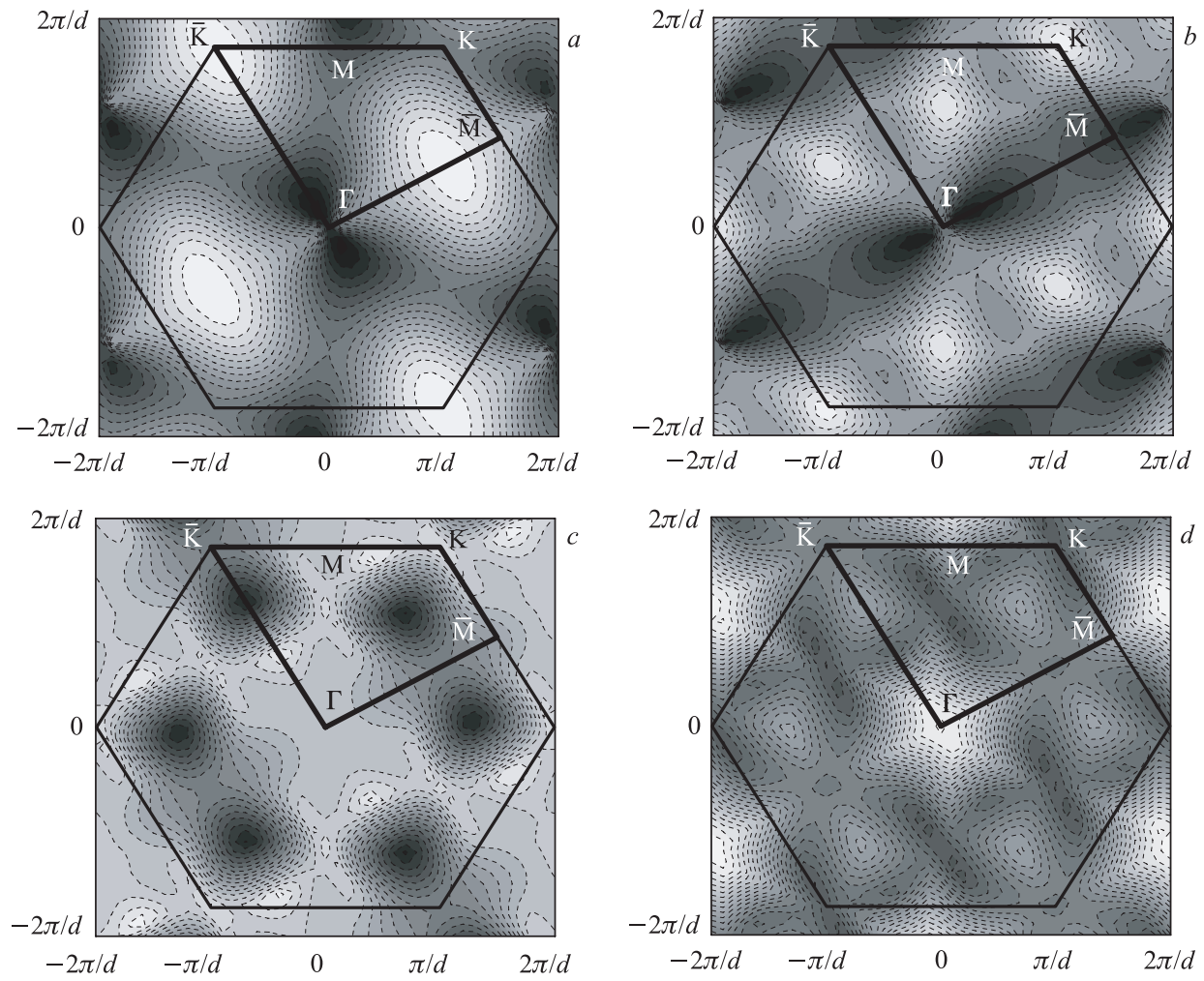

Fig. 3. Contour plots of HCPCF cladding dispersion surface, corresponding to the first $(a)$, the second $(b)$, the third $(c)$ and the fourth eigenvalue $(d)$ of the eigenmodal problem. The magnitude of the wave number is denoted by the grey scale. Dashed lines correspond to the isofrequencies. The Brillouin zone is shown by the solid line (the irreducible part of it is shown by the thick line) 


\section{DISPERSION DIAGRAMS AND HCPCF MODES CALCULATION}

Pioneering the use of the same plane wave method modification, we solved the dispersion equation for the HCPCF, represented as a superlattice, assuming that modes of the neighbor fibers (cores of the neighbor supercells) of this structure are phased-in and exert no influence on each other.

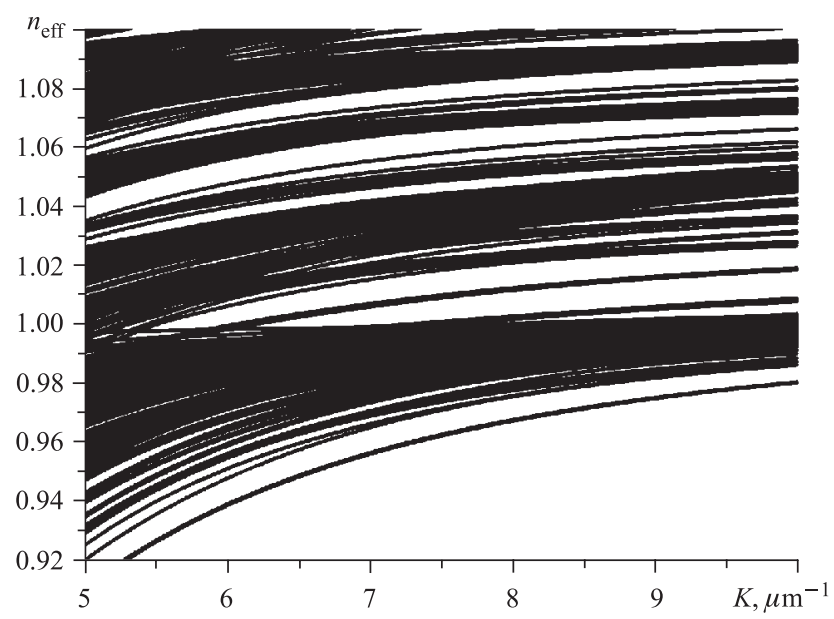

Fig. 4. HCPCF modal dispersion diagram. Each line corresponds to a certain mode

Thus, instead of permitted and forbidden areas, lines appear on the dispersion diagram of the HCPCF (Fig. 4). Each line corresponds to a certain fiber mode, which can be guided through the HCPCF only within the cladding band gap. This condition is easily checked by imposing the dispersion diagram of HCPCF on that of its cladding (Fig. 5). The field of the modal lines, corresponding to white areas (cladding band gaps), is confined within the core due to the band gap effect.

We are interested in transmitting the localized modes, i.e., those which have their field confined within the core. Due to the fact that the core of the fibers concerned is hollow (air-filled) the mode propagates through the air. Thus, one can draw a conclusion that the effective refraction index in the waveguiding regimes is less than unity. This distinction allows one to separate volume waves from localized and surface modes when analyzing the dispersion diagram.

The modal structure of the fiber is complicated and there are multiple crossings of the modal characteristics on the dispersion diagram. It means that once the necessary mode is excited, there is still a probability of its energy being transmitted to a neighbor surface mode. In order to avoid such situations, we have studied the points of HCPCF modal lines crossing. Calculating the eigenvectors of the problem yields the modal field transversal distribution and allows one to track the modal behavior, when the system parameters are changing. Thus, Fig. 5 presents an example of how the modes exchange places in the HCPCF modal spectrum. Our treatment is convenient as the fiber modes in this approach are not bound and, consequently, their field profiles do not change along the modal lines, apart from the crossing points, where it turns into a superposition of two corresponding modal fields. 


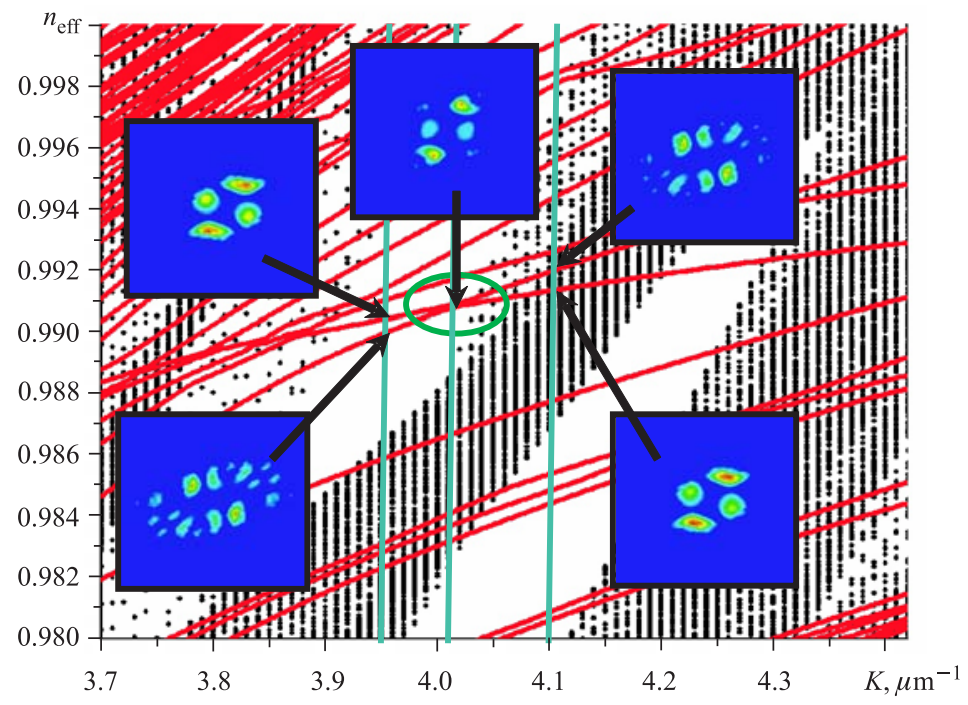

Fig. 5. HCPCF dispersion diagram. Grey areas represent the permitted areas of the cladding structure, the white ones correspond to band gaps. HCPCF core modes are shown by black solid lines. The incuts illustrate the behavior of modal fields in the vicinity of the modal dispersion characteristics intersection point. Band gaps are shown by white areas, the shaded areas correspond to HCPCF cladding permitted bands. Red solid lines denote the HCPCF core modes. The incuts show the corresponding modal Poynting vector transversal distribution

\section{CONCLUSION}

In this paper we have demonstrated a numerical model for hollow-core modal spectra calculations which is based on the plane wave decomposition. This model was used for calculations of band gaps structure for fiber cladding and for core modes of the diamondshaped and Kagomi-lattice hollow-core photonic crystal fibers. The dispersion characteristics of modes in allowed and forbidden bands were shown. The points of the dispersion characteristics intersections were analyzed. The dispersion properties of hollow-core photonic crystal fibers (made of soft optical glass) with diamond-shaped, small-area, and large-area cores were studied.

Acknowledgements. The work was supported by CRDF REC-006 and the Dynasty Foundation.

\section{REFERENCES}

1. Joannopoulos J. D., Meade R. D., Winn J. N. Photonic Crystals: Molding the Flow of Light. Princeton: Princeton University Press, 1995.

2. Knight J. C. et al. Photonic Band Gap Guidance in Optical Fibres // Science. 1998. V.282. P. 14761478. 
3. Barkou S.E., Broeng J., Bjarklev A. Silica/Air Photonic Crystal Fibre Design That Permits Waveguiding by a True Photonic Bandgap Effect // Opt. Lett. 1999. V. 24. P. 46-48.

4. Broeng J. et al. Waveguidance by the Photonic Bandgap Effect in Optical Fibres // J. Opt. A: Pure Appl. Opt. 1999. V.1. P. 477-482.

5. Konorov S. O. et al. Waveguide Modes of Electromagnetic Radiation in Hollow-Core Microstructure and Photonic-Crystal Fibers // JETP. 2003. V.96, No. 5. P. 857-869.

6. Galea A. D. et al. Selective Mode Excitation in Hollow-Core Photonic Crystal Fiber // Opt. Lett. 2005. V. 30, No. 7. P. 717-719.

7. Luan F. et al. Femtosecond Soliton Pulse Delivery at $800 \mathrm{~nm}$ Wavelength in Hollow-Core Photonic Bandgap Fibers // Opt. Express. 2005. V. 12. P. 835-840.

8. Birks T. A. et al. Ultimate Low Loss of Hollow-Core Photonic Crystal Fibers // Opt. Express. 2004. V. 10. P. 239-244. 